\section{Anticoagulation during ECMO: The Past, Present and Future}

\section{Abstract}

In recent years, many medical centres started to use extracorporeal membrane oxygenator (ECMO) support as the last treatment option for critically ill patients when the conventional therapy fails. There are significant improvements in ECMO management since the early days of its application. In this report, we aimed to review several aspects in this complex treatment modality. One of the main areas reviewed in this article will be the anticoagulation strategy used during ECMO support and the tests utilized for monitoring coagulation parameters. Additionally, technologies used in ECMO circuits and new advances in the field were reviewed.

Keywords: Extracorporeal membrane oxygenation; ECMO cannulation; Anticoagulation

Received: April 26, 2018; Accepted: May 08, 2018; Published: May 15, 2018

\begin{abstract}
Sule Doymaz*
Division of Pediatric Critical Care, Department of Pediatrics, State University of New York, USA
\end{abstract}

\section{*Corresponding author: Sule Doymaz}

sule.doymaz@downstate.edu

Division of Pediatric Critical Care, Department of Pediatrics, State University of New York, USA.

Tel: (718) 270-1625

Citation: Doymaz S. Anticoagulation during ECMO: The Past, Present and Future. J Intensive \& Crit Care Vol.4 No.2:12

\section{Introduction}

ECMO can be a lifesaving treatment option for critically ill patients when the conventional methods cannot deliver the intended results. ECMO first started to be utilized during the 1970s. However, in the initial trials, ECMO was fraught with high rate of mortality and consequently only limited number of centres adapted the system [1].

The initial high rates of mortality and less than optimal success rates negatively influenced the wider acceptance of ECMO until the H1N1 outbreak of 2009. During the H1N1 outbreak, ECMO proved to be useful for many critically ill patients and fell back in favour. This period was a turning point for ECMO based treatment methods that were adopted in many tertiary healthcare centres since. The new advances in ECMO technologies and the development of more efficient circuits have also played a significant role in the wider acceptance of this methodology [2].

Currently, many centres have adopted ECMO as a last-resort of therapy for critically ill patients who need respiratory and/or cardiovascular support. Many others are on their way to bring the system on board. Information and statistics on the current working ECMO systems are available through the Extracorporeal Life Support Organization (ELSO) Registry. The ELSO registry was instituted in 1999 and is widely used by ECMO centres since. The ELSO Registry collects and analyses the data from the ECMO centres and makes it available for the interested parties. Currently, ECMO support is the last- resort of treatment for many critically ill patients and list of its applications continue to grow.
The current conditions where ECMO is indicated are updated on the ELSO Registry website [3].

In recent years, Extracorporeal Cardiopulmonary Resuscitation (ECPR) has been employed in patients with respiratory and cardiac arrests in pre-hospitalization and emergency settings with more than gratifying results. [4].

Many of these satisfactory outcomes could be linked to the new advances in ECMO technologies and the ever-increasing ECMO experience in healthcare centres by healthcare teams using this modality. It appears that ECPR will be more commonly used in the near future for various medical emergencies because of the successful outcomes the system produces. It is not surprising to forecast that the technological advances will bring more advanced ECMO circuits to the practice field that in turn will help to bring better outcome for patients [5-7].

\section{Types of ECMO}

Currently there are two types of ECMO support used in critical care practice. These are veno-venous (VV) and veno-arterial (VA) ECMO, selected depending on the underlying condition. VV ECMO is used for patients with respiratory failure where ventilation and oxygenation is supported through artificial oxygenator bypassing the patients' lung. Through a cannula placed in one of the major veins, the deoxygenated blood is transferred to the ECMO circuit and passed through oxygenator and returned to venous circulatory system of the patient. There is no cardiac support in VV ECMO (Figure 1). 
The VA ECMO is used for patients who need respiratory and circulatory support. In VA ECMO, the deoxygenated blood of the patient is circulated through a pump and the heart is bypassed. Blood is oxygenated through an artificial oxygenator and returns back to the arterial circulation of the patient. In this modality, two major cannulas are placed in a major vein and artery (Figure 2) $[8,9]$.

There is no consensus on selection criteria among the ECMO centers. We need more research in this area to obtain better evidence to guide us on ECMO type selection.

\section{ECMO Oxygenator}

The oxygenator is an important component of the ECMO circuit. It functions as an artificial lung removing carbon dioxide and oxygenating blood. Early oxygenators were made of silicon materials whereas in recent years, hallow fiber oxygenators are mostly utilized [10]. Compared to silicon oxygenators, hallow fiber oxygenators have a shorter prime time with lower prime volume that makes them superior. Additionally, HF oxygenators have lower trans-membrane pressure and the drop in pressure occurs less frequently [11].
Another advantage of HF oxygenators is the possibility of surface coating that leads to less clot formation. However, leakage of plasma is a significant problem noticed with this type of oxygenators. Implementation of hallow fiber micro porous technology using the patented Membrana ${ }^{\circledR}$ polymethylpentene (PMP) hollow fiber seems to alleviate leakage of plasma in these oxygenators [12]. Consequently, long-term usage seems to be possible with these new devices and many neonatal ECMO centers are currently using the devices equipped with PMP membrane oxygenators [13].

\section{ECMO Pumps}

Mainly, there are 2 types of pumps that are used in ECMO circuits: Roller pumps and centrifugal pumps. Roller pumps were used for many years and currently are increasingly being replaced by centrifugal pumps. Initial studies comparing the $1^{\text {st }}$ generation centrifugal pumps to roller pumps were generally inconclusive. In recent years, new generation of centrifugal pumps with magnetic levitations were developed and are as efficient as roller pumps and more effective in returning end-organ functions. Also, the centrifugal pumps with magnetic levitations seem to provide better short and long-term survival rates [14].
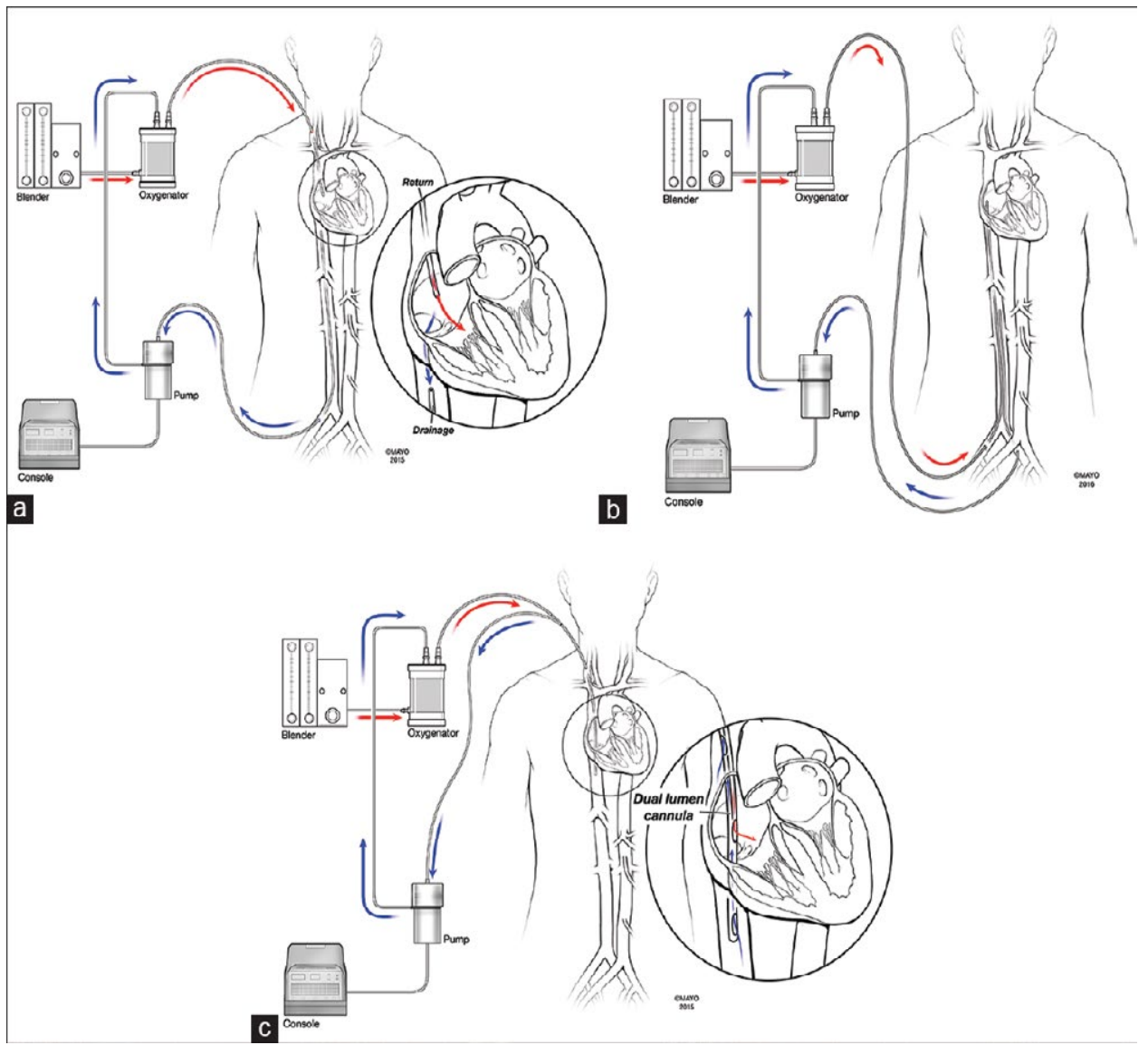

Figure 1 VV ECMO cannulation types: (a) Drainage cannula tip is in inferior vena cava and the return cannula is in the right atrium. (b) Tip of the drainage cannula is in the infra hepatic inferior vena cava and the return cannula tip is in the right atrium. (c) Venous cannula with bicaval drainage ports and a return port directs the oxygenated blood toward the tricuspid valve [8]. 


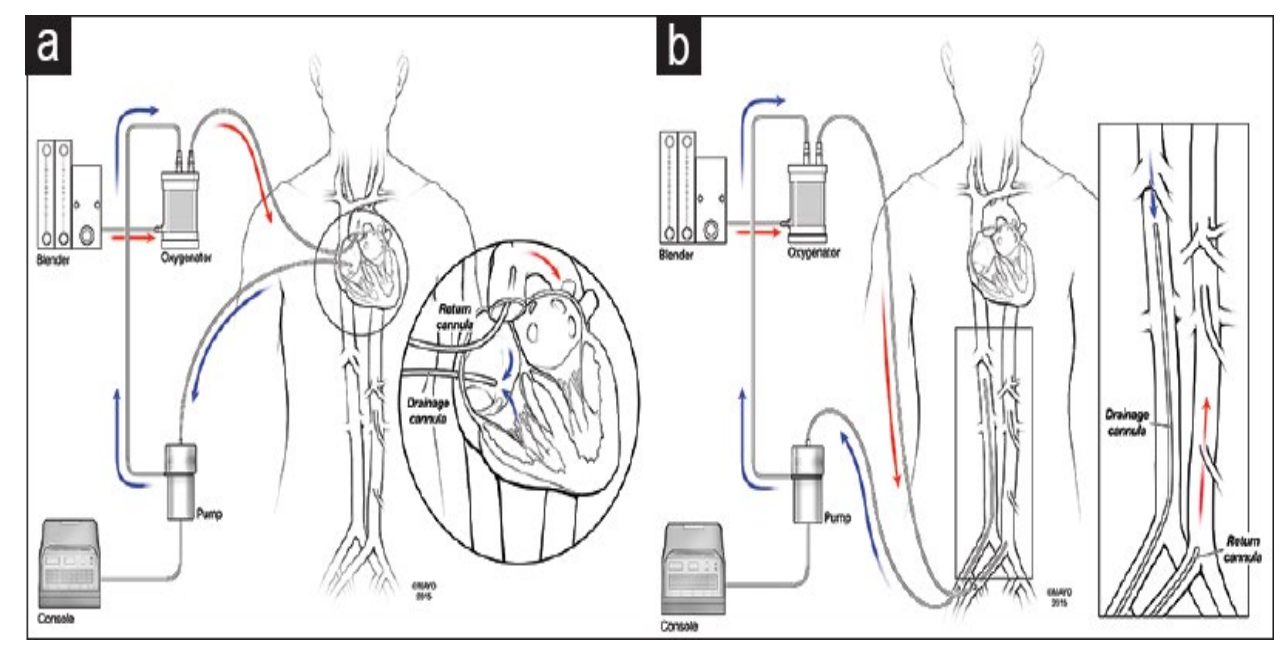

Figure 2 Central VA ECMO cannulation: (a) Venous cannula draining the blood from right atrium and arterial cannula returning the blood to aorta, (b) Peripheral VA ECMO cannulation: Venous cannula draining the blood from femoral vein and arterial cannula returning the blood to femoral artery [8].

Roller pumps can be partially or totally occlusive whereas centrifugal pumps are non-occlusive. Roller pumps push the blood forward by squeezing the raceway. The flow is determined by the stroke volume and rpm. In centrifugal pumps, flow is dependent on the preload and afterload. In these types of pumps resistance inversely affects the flow rate. In roller pumps blood in the tubing is being compressed in the raceway and this causes mechanical trauma to the blood. This can result in hemolysis and activation of the immune system. Inlet and outlet pressures should be monitored closely to prevent cavitation, heat production and hemolysis when using centrifugal pumps. Hemolysis is the least concern with centrifugal pumps with magnetic levitation because there is no stress on the blood. Blood is moved by the magnetic forces generated by the pump motor.

\section{Monitoring Patients on ECMO}

ECMO is an extremely invasive treatment modality and consequently close monitoring is required for achieving the best outcome. The monitoring is carried out both for the patients and the circuit because any deficiency in the function of the circuit may result in mortality. Complications related to the system could be originating from any part of the circuit. Clot formation, air emboli, oxygenator/pump failure and issues related to cannulation could be listed as circuit complications. For patients, bleeding, infection and hemolysis are listed as the most commonly encountered complications [15].

During ECMO support, bleeding and thrombotic complications carry the most significant risk for morbidity and mortality. According to the ELSO Registry Report, the rate of complication of clot formation resulting in the circuit replacement is about $20 \%[16]$.

Another serious complication of ECMO is bleeding. It can occur due to factors inherent to the patient's underlying problems or to the anticoagulation medications used during the treatment.
Therefore, patients who are under consideration for ECMO support should carefully be evaluated for the risk of bleeding. Bleeding can occur in any part of the body but intracranial bleeding is the most dreaded. In a previous study in 32 neonatal patients on ECMO support for persistent pulmonary hypertension, we reported a rate of intracranial hemorrhage of 34\% [17].

\section{Why Do We Need Anticoagulation during ECMO?}

ECMO support continues to be the last hope for survival in certain critically ill patients. However, despite tremendous advances in ECMO circuit technology in recent years, we are still far from having a satisfactory circuit design where no anticoagulation is needed. Proteins in blood accumulate on the circuit membranes causing membrane fouling leading to the activation of the coagulation cascade and creating the risk for platelet and leukocytes aggregation to the membrane surfaces. Once the enzymes are activated, FXII, complement components, kinin/kallikrein and fibrinogens are cleaved switching on the coagulation cascade and eventually lead to thrombus formation. Currently, heparin coated membranes are utilized in many ECMO oxygenators however this solution does not seem to eliminate the risk of coagulation completely [18]. In experimental animal studies, adding nitric oxide releasing polymers to the ECMO circuit improved the platelet activation and consumption process. Nitric oxide is released from the endothelium and prevents platelet aggregation [19]. Adding direct thrombin inhibitor such as argatroban to the ECMO circuit coated with NO releasing material further improved the coagulation process in a rabbit model [20].

\section{Management and Monitoring of Anticoagulation}

Since the formation of thrombus during the ECMO process is 
not induced by a single factor, the solution to the problem is challenging. The ELSO Registry has published a guideline for anticoagulation with the help of the experts in the field [21].

This report provides general guidelines for ECMO centers in prevention of coagulation during the ECMO procedures. However emphasis is made that each patient receives a specific individualized anticoagulation regimen. Currently, complications linked to anticoagulation regimens seem to be on the rise especially in the young and pediatric patients. This trend is observed worldwide underscoring the need for addressing anti coagulation measures in ECMO practices [22].

For the reason listed above, ECMO patients need to be monitored and evaluated closely due to the complications related to coagulation and anticoagulation regimens. Before initiation of ECMO support it is important to obtain complete blood count, PT/INR, APTT, fibrinogen, D-dimer, activated clotting time (ACT), anti-thrombi activity (AT), thromboelastography (TEG), thromboelastometry (ROTEM). The results will provide a baseline on the patient and help to assess the coagulation parameters later in the ECMO course.

For decades un-fractionated heparin (UNFH) has been used widely as the preferred anti-coagulant in many ECMO centers. Direct thrombin inhibitors can be considered. However they are not a viable alternative to heparin due to the lack of monitoring methods and antidotes [23].

Heparin reacts with anti-thrombin (AT) and tissue factor pathway inhibitor (TFPI). UNFH bound AT is 1000 more active in preventing thrombus formation than unbound-AT. UNFH also increases the affinity of TFPI to factor $\mathrm{Xa}$ at least 2 to 4 times and reversibly inactivates the function of Factor Xa. Afterwards, the formed complex of Factor Xa-TFPI by the help of UNFH inhibits the Factor VIla-tissue factor complex. Generally heparin is given intravenously with 50 to $100 \mathrm{U} / \mathrm{kg}$ as an initial bolus and when activated coagulation time (ACT) reaches to $300 \mathrm{~s}$, heparin doses are adjusted to 7.5 to $20 \mathrm{U} / \mathrm{kg}$ per $\mathrm{h}$ for adults. Neonates have lower levels of anti-thrombin in their body for this reason their initial heparin doses are higher. ACT testing is a non-specific point of care assessment method used since the 1970s when ECMO first started to be utilized. ACT is still widely used for dosage adjustment of the heparin drip in many ECMO centers. During the heparin infusion, ACT levels should be kept between 180 and $220 \mathrm{~s}$ [24]. In ACT testing, whole blood specimen sample is used many factors in the blood and thrombocyte activity of blood influence the result of the test. The ACT testing is not a specific test for assessing heparin activity. In recent years, anti-factor $\mathrm{Xa}$ assay was evaluated as a possible replacement for ACT and APTT tests. The current view is that anti factor Xa test might provide superior results in adjusting heparin doses that those given by ACT and aPTT $[25,26]$.

In many ECMO centers, anti-factor Xa testing is incorporated in their anti-coagulation protocols. The widely accepted anti-factor Xa value ranges between 0.3 and $0.7 \mathrm{IU} / \mathrm{ml}$. The availability of many different anti-factor $\mathrm{Xa}$ assays on the market and their variability in assessing heparin levels poses a potential problem in this field. Consequently, a wider acceptance of this test is largely hindered by the lack of standardization. It is our view that as the experience with this test increases and adequate standardization is reached, a wider acceptance and utilization of the assay will be attained.

Another test used in monitoring the heparin dose in ECMO patient is aPTT assay. Generally, maintenance of aPTT levels at 1.5 to 2.5 is the suggested level for anticoagulation regimens. aPTT concentration in blood varies according to the age of the subjects. Therefore levels differ between adults and pediatric patients. In addition different assays are available and make it difficult to utilize aPTT as the sole monitoring modality in anticoagulation for ECMO patients.

Thromboelastography (TEG) and rotational thromboelastometry (ROTEM) test elastic properties of the whole blood during coagulation. These assays are commonly used in evaluating the coagulation status of the patients during major cardiac and hepatic surgeries and traumas. The assays provide a rapid assessment of the need for blood transfusion for patients. However, the current lack of standardizations has hindered the wider adoption of the methodology. More detailed studies are needed for these assays to be useful in monitoring patients on ECMO support.

Fibrinogen levels and platelet counts also need to be monitored closely during ECMO support. Platelet counts more than 80.000 cells $/ \mathrm{mm}^{3}$ and fibrinogen levels above $100 \mathrm{~g} / \mathrm{dL}$ are acceptable during ECMO support as per the current guidelines. However, coagulation management should be individualized based on the patients' requirements [21]. In our previous study, low fibrinogen level (mean of $115 \pm 13$ vs. higher level of $175.0 \pm 74.8$, $\mathrm{p}=0.009$ ) and low platelet count (mean of $37.4 \pm 18.3 \mathrm{vs}$. higher level of $116.4 \pm 18.9, p=0.005$ ) were identified as risk factors for developing intracranial bleeding in the neonatal population with persistent pulmonary hypertension [17]. Close monitoring of these parameters and maintaining fibrinogen levels and platelet counts within normal range may prevent this mostly fatal complication.

\section{What is in the Future?}

In recent years, different studies are looking at the various design of the histocompatible ECMO membrane. Coating the membrane surfaces with synthetic and natural polymers and endothelialization of the membrane surfaces are two current research areas. In a recent study, polymer brushes grafted with the technique of single electron-transfer living radical polymerization (SET-LRP) was used on polymethylpentene types of membranes. After exposure to human plasma, it was noticed that re-calcification time was significantly prolonged as a result of delay in coagulation. This method significantly prevented the binding of the platelets and leukocytes on the surfaces [18].

A healthy vascular endothelial tissue plays an important role in the coagulation process. The ultimate aim of membrane technologies on ECMO circuits should be to mimic the healthy endothelial tissue as much as possible. Only after then, one can expect to overcome the myriad of coagulation problems experienced during ECMO treatment. Recent advances in tissue 
engineering promise to solve some of the issues encountered in this field. Accordingly, endothelial cells could be seeded into the polypropylene membranes and after the attachment the cells could show in vitro proliferations on the membranes [27].

Another study showed that membrane surfaces covered with titanium dioxide would facilitate attachment of endothelial cells to allow the development of mono-layered endothelium, which does not seem to produce any adverse effects on the oxygenation process [28].

New durable and biocompatible ECMO pump system was tested successfully on a large animal model with no clot formation around the bearing [29].

These exciting studies foster the hope that the future of ECMO practices will be much less complicated and safer than they are today. However, the translation from animal experimentation to human use is not a short and quick road.

\section{Conclusion}

ECMO continues to be the hope for survival for many critically

\section{References}

1 Gibbon Jr JH (1954) Application of a mechanical heart and lung apparatus to cardiac surgery. Minn Med pp: 171-185.

2 Schmidt M, Hodgson C, Combes A (2015) Extracorporeal gas exchange for acute respiratory failure in adult patients: A systematic review. Crit Care 19: 99.

3 https://www.elso.org/Registry/SupportDocuments.aspx

4 Chen YS, Lin JW, Yu HY, Ko WJ, Jerng JS, et al. (2008) Cardiopulmonary resuscitation with assisted extracorporeal life-support versus conventional cardiopulmonary resuscitation in adults with inhospital cardiac arrest: An observational study and propensity analysis. Lancet 372: 554-561.

5 Lamhaut L, Jouffroy R, Soldan M, Phillipe P, Deluze T, et al. (2013) Safety and feasibility of prehospital extra corporeal life support implementation by non-surgeons for out-of-hospital refractory cardiac arrest. Resuscitation 84: 1525-1529.

6 Firstenberg MS, Galloway J, Abel E, Papadimos TJ, Burcham P, et al. (2011) Extra-corporeal circulatory support: A resurgence of a lifesaving therapy in the digital information age. Surg Curr Res 1: 103.

7 Bartlett R (2016) ECMO: The next ten years. Egypt J Crit Care Med pp: 7-10.

8 Jayaraman AL, Cormican D, Shah P, Ramakrishna H (2017) Cannulation strategies in adult veno-arterial and veno-venous extracorporeal membrane oxygenation: Techniques, limitations and special considerations. Ann Card Anaesth 20: S11-S18.

9 Fletcher K, Chapman R, Keene S, Perinatol S (2018) An overview of medical ECMO for neonates 42: 68-79.

10 Palanzo D, Qiu F, Baer L, Clark JB, Myers JL, et al. (2010) Evolution of the ECLS circuitry 11: 869-873.

11 Mejak B, Giacomuzzi C, Heller C, You X, Ungerleider R, et al. ill patients. However, ECMO treatment is not without its own drawbacks. These drawbacks not only stem from the nature of the treatment modality, which is a highly invasive procedure, but also from the fact that these patients are critically ill and do not respond to conventional medical treatment. Another significant potential problem associated with ECMO treatment is the risk of blood coagulation and the need for anti-coagulation during the treatment. Anticoagulation therapy has risks of serious bleeding complications. The experience on anticoagulation medications for ECMO patients and the technology on this area are relatively limited. Despite the hopeful recent technological advances on ECMO circuits as well as on the large number of publications on ever-widening ECMO practices, there is still a long way to reach optimum care. It is our hope that as experiences on various ECMO practices are collected, published, and analyzed, a unified treatment approach will emerge for similar cases and the differences among the medical centers will be minimized. Also, with the technological developments of ECMO circuits more efficient, user friendly and less error-prone instruments will be at the disposal of critical care professionals to reach ever increasing quality metrics in care.

(2007) Retrospective analysis comparing hollow fiber and silicone membrane oxygenators for neonates on ECMO. J Extra Corpor Technol 39: 71-74.

12 Thiara AP, Hoel TN, Kristiansen F, Karlsen HM, Fiane AE, et al. (2007) Evaluation of oxygenators and centrifugal pumps for long-term pediatric extracorporeal membrane oxygenation. Perfusion 22: 323326.

13 Lawson S, Ellis C, Butler K, McRobb C, Mejak B (2011) Neonatal extracorporeal membrane oxygenation devices, techniques and team roles: 2011 survey results of the United States' extracorporeal life support organization centers. J Extra Corpor Technol 43: 236244.

14 Luciani GB, Hoxha S, Torre S, Rungatscher A, Menon T, et al. (2016) Improved outcome of cardiac extracorporeal membrane oxygenation in infants and children using magnetic levitation centrifugal pumps. Artif Organs, pp: 27-33.

15 Butt W, Heard M, Peek GJ (2013) Clinical management of the extracorporeal membrane oxygenation circuit. Pediatr Crit Care Med 14: S13-S9.

16 Extracorporeal Life Support Organization (2013) Registry report. Ann Arbor: University of Michigan.

17 Doymaz S, Zinger M, Sweberg T (2015) Risk factors associated with intracranial hemorrhage in neonates with persistent pulmonary hypertension on ECMO. J Intensive Care 3: 6 .

18 Obstals F, Vorobii M, Riedel T, Bruns M, Singh S, et al. (2018) Improving hemocompatibility of membranes for extracorporeal membrane oxygenators by grafting nonthrombogenic polymer brushes. Macromol Biosci.

19 Annich GM, Meinhardt JP, Mowery KA, Ashton BA, Merz SI, et al (2000) Reduced platelet activation and thrombosis in extracorporeal circuits coated with nitric oxide release polymers. Crit Care Med 28: 915. 
20 Terry C, Elizabeth M, Brisboisb J, Margaux AM, Gail EZ, et al. (2014) The effect of a polyurethane coating incorporating both a thrombin inhibitor and nitric oxide on hemocompatibility in extracorporeal circulation. Biomaterials 35: 7271-7285.

21 (2014) ELSO anticoagulation guidelines 2014.

22 Stocker CF, Horton SB (2016) Perfusion anticoagulation strategies and difficulties in neonatal and paediatric extracorporeal membrane oxygenation (ECMO). Perfusion 31: 95-102.

23 Cho HJ, Kim DW, Kim GS, Jeong IS (2017) Anticoagulation therapy during extracorporeal membrane oxygenator support in pediatric patients. Med J 53: 110-117.

24 Kamdar A, Rintoul N, Raffini L (2018) Anticoagulation in neonatal ECMO. Semin Perinatol 42: 122-128.

25 Liveris A, Bello RA, Friedmann P, Duffy MA, Manwani D, et al. (2014) Anti-factor $\mathrm{Xa}$ assay is a superior correlate of heparin dose than activated partial thromboplastin time or activated clotting time in pediatric extracorporeal membrane oxygenation. Pediatr Crit Care Med 15: e72-79.

26 O'Meara LC, Alten JA, Goldberg KG, Timpa JG, Phillips J, et al. (2015) $S$ Anti-xa directed protocol for anticoagulation management in children supported with extracorporeal membrane oxygenation. ASAIO J 61: 339-344.

27 Cornelissen CG, Dietrich M, Gromann K, Frese J, Krueger S, et al. (2013) Fibronectin coating of oxygenator membranes enhances endothelial cell attachment. Biomed Eng Online 12: 7.

28 Pflaum M, Kühn-Kauffeldt M, Schmeckebier S, Dipresa D, Chauhan $\mathrm{K}$, et al. (2017) Endothelialization and characterization of titanium dioxide-coated gas-exchange membranes for application in the bioartificial lung. Acta Biomater 50: 510-521.

29 Lizuka K, Katagiri N, Takewa Y, Tsukiya T, Mizuno T, et al. (2018) E1 evaluation of the novel centrifugal pump, CAPIOX SL, in chronic large animal experiments. Artif Organs. 